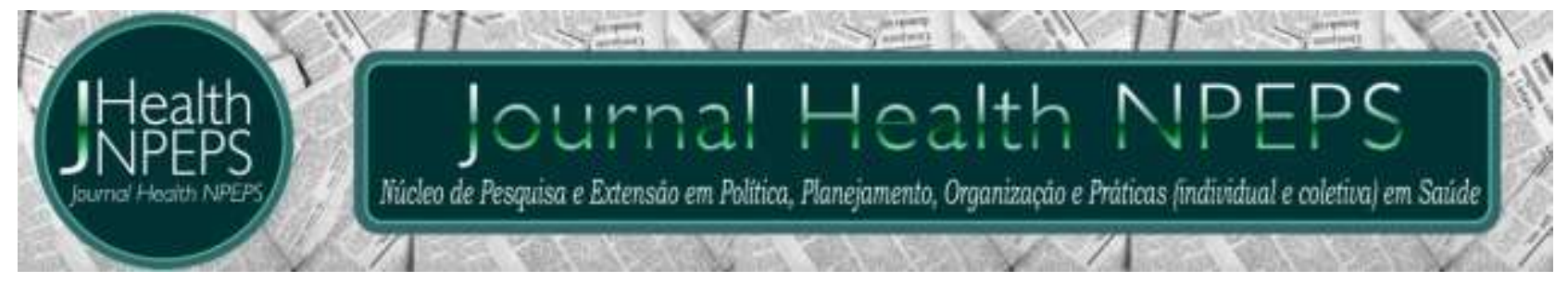

http://dx.doi.org/10.30681/252610102758

ARTIGO ORIGINAL

\title{
Relación del malestar emocional y el consumo de alcohol en adolescentes
}

Relationship of emotional distress and alcohol consumption in adolescents

Relação do sofrimento emocional e o consumo de álcool em adolescentes

\section{Juan Yovani Telumbre Terrero ${ }^{1}$, Manuel Antonio López Cisneros ${ }^{2}$, Laura Edith Peralta Telumbre ${ }^{3}$, Oscar David Velázquez Linares ${ }^{4}$, Reyna Torres Obregón ${ }^{5}$, Santiaga Enriqueta Esparza Almanza ${ }^{6}$}

\section{RESUMEN}

Objetivo: determinar la relación y el efecto del malestar emocional en el consumo de alcohol de los adolescentes. Método: estudio cuantitativo transversal, descriptivo y predictivo, llevado a cabo en 200 adolescentes de ambos sexos de una institución pública de educación secundaria de Ciudad del Carmen Campeche, México; durante el mes enero de 2018. Se utilizó una cedula de datos sociodemográficos y antecedentes del consumo de alcohol y el Cuestionario de Factores de Riesgo y de Protección para el Consumo de Drogas. Los datos se procesaron en el programa SPSS a través de la estadística descriptiva e inferencial. Resultados: $19.5 \%$ de los adolescentes manifestaron haber ingerido alguna bebida alcohol en algún momento de su vida y el $14 \%$ en el último año. $64 \%$ presenta malestar emocional, de los cuales destaca la tristeza, alegría, aburrimiento y pensamientos repetitivos. Se observó una relación positiva $(r s=.473, p=.003)$ y efecto $(p=.001, f=10.787, g l=1)$ del malestar emocional con el consumo de alcohol. Conclusión: se pudo identificar que el malestar emocional está asociado a la ingesta de alcohol, esto como una forma de mitigar ese conjunto de emociones y sentimientos negativas y/o positivas característicos de esta etapa.

Descriptores: Adolescente; Consumo de Bebidas Alcohólicas; Emociones; Síntomas Afectivos.

\footnotetext{
${ }^{1}$ Enfermero. Maestro em Ciencias de Enfermería. Profesor Investigador de Tiempo Completo de la Universidad Autónoma del Carmen. Ciudad del Carmen Campeche, México. E-mail: jytt_@hotmail.com. ORCID ID: https://orcid.org/0000-0002-8695-7924. Autor principal - Dirección de envío: Facultad de Ciencias de la Salud. Campus III. Universidad Autónoma del Carmen. Av. Central s/n. Esq. Fracc. Mundo Maya. CP. 24115. Ciudad del Carmen Campeche. Tel. 9383811 018. Ext. 2301.

${ }^{2}$ Enfermero. Doctor em Ciencias de Enfermería. Profesor Investigador de Tiempo Completo de la Universidad Autónoma del Carmen. Ciudad del Carmen Campeche, México. E-mail: mlopez@pampano.unacar.mx ORCID ID: https://orcid.org/0000-0002-9384-5752.

${ }^{3}$ Enfermera. Licenciada en Enfermería. Universidad Autónoma de Guerrero. Chilpancingo de los Bravo Guerrero, México. E-mail: edith1515_@hotmail.com ORCID ID: https://orcid.org/0000-0002-8623-7062.

${ }^{4}$ Enfermero. Licenciado en Enfermería. Universidad Autónoma del Carmen. Ciudad del Carmen Campeche, México. E-mail: david.91811vl@gmail.com ORCID ID: https://orcid.org/0000-0002-8079-6023

${ }^{5}$ Enfermera. Doctora em Ciencias de Enfermería. Profesor Investigador de Tiempo Completo de la Universidad Autónoma de Coahuila. Unidad Saltillo, México. E-mail: reyna_89_12@hotmail.com ORCID ID: https://orcid.org/00000003-3546-6970.

${ }^{6}$ Enfermera. Maestra em Ciencias de Enfermería. Profesor Investigador de Tiempo Completo de la Universidad Autónoma de Nuevo León, México. E-mail: sesparza54@yahoo.com.mx ORCID ID: https://orcid.org/0000-0003-42542791.
} 


\section{ABSTRACT}

Objective: determine the relationship and effect of emotional distress on adolescents' alcohol consumption. Method: quantitative, cross-sectional, descriptive and predictive study was conducted in 200 middle school adolescents of both sexes in Ciudad del Carmen, Campeche, Mexico; during the month of January 2018. A sociodemographic data and alcohol consumption history and the Questionnaire on Risk Factors and Protection for Drug Use was used. Results: $19.5 \%$ of the adolescents claimed to have ingested an alcoholic beverage at some time in their life and 14\% in the last year. 64\% present emotional discomfort, of which sadness, happiness, boredom and repetitive thoughts stand out. A positive relationship was observed $(r s=.473, p=.003)$ and effect $(p=.001, f=10.787, g l=1)$ of emotional distress with alcohol consumption. Conclusion: it was possible to identify that the emotional distress is associated with alcohol consumption, this as a way to mitigate that set of negative and/or positive emotions and feelings characteristics of this stage.

Descriptors: Adolescent; Alcohol Drinking; Emotions; Affective Symptoms.

\section{RESUMO}

Objetivo: determinar a relação e o efeito do desconforto emocional no consumo de álcool dos adolescentes. Método: estudo quantitativo de corte transversal, descritivo e preditivo, junto a 200 adolescentes de ambos sexos de uma instituição pública de ensino fundamental e médio da Ciudad del Carmen, Campeche, México, durante o mês de janeiro de 2018. Foi utilizado um questionário com dados sociodemográficos e uma história de álcool e o Questionário de Fatores de Risco e de Proteção para o Consumo de Drogas. Resultados: $19,5 \%$ dos adolescentes manifestaram ter ingerido alguma bebida alcoólica em algum momento da sua vida e o $14 \%$ no último ano. $64 \%$ apresenta desconforto emocional, dos quais destaca a tristeza, alegria, tédio e pensamentos repetitivos. Observou-se uma relação positiva $(r s=.473, p=.003)$ e efeito $(p=.001$, $f=10,787, g l=1$ ) do desconforto emocional com o consumo de álcool. Conclusão: foi possível identificar que o desconforto emocional está associado ao consumo de álcool, isso como uma forma de mitigar esse conjunto de emoções e sentimentos negativos e/ou positivos caraterísticos dessa etapa.

Descritores: Adolescente; Consumo de Bebidas Alcoólicas; Emoções; Sintomas Afetivos.

\section{INTRODUCCIÓN}

El uso y/o abuso de alcohol representa un problema de salud pública mundial dado que puede dañar la vida del bebedor, quebrantar a su familia y deteriorar el tejido social con altas implicaciones económicas y sanitarias. Además, es considerado uno de los principales factores de riesgo de muerte prematura, discapacidad, trastornos neuropsiquiátricos, enfermedades cardiovasculares, cirrosis hepática y diversos tipos de cáncer ${ }^{1}$.

En las Américas, el consumo de alcohol es de aproximadamente un $60 \%$ con un patrón de consumo peligroso para la salud, asociado a diversos daños a la salud, que incluyen más de 200 condiciones, violencia doméstica, pérdida de productividad, entre otros $^{2}$. Se ha documentado que en los últimos cinco años han aumentado los episodios 
de consumo excesivo de alcohol, pasando de $4.6 \%$ a un $13 \%$ en el caso de las mujeres y del $17.9 \%$ al $29.4 \%$ en los hombres. Específicamente en adolescentes se identificó un patrón de menor frecuencia, pero alta ingesta en una sola ocasión, iniciado su consumo antes de los 14 años de edad ${ }^{3}$.

En México se reporta que la prevalencia total de consumo de alcohol en los estudiantes de secundaria y bachillerato es de $53.2 \%$; $54 \%$ en los hombres y $52.5 \%$ en las mujeres. Por su parte, el $35.5 \%$ de los estudiantes consumieron alcohol en el último año, en los hombres este consumo fue de $24.3 \%$ en secundaria y $56.5 \%$ en bachillerato, en las mujeres fue de $24 \%$ en secundaria y $52.1 \%$ en bachillerato. El consumo excesivo de alcohol es de $14.5 \%$ en los estudiantes, $15.7 \%$ para los hombres y el $13.3 \%$ para las mujeres. La bebida de mayor consumo es la cerveza, seguido de los destilados, las bebidas preparadas en lata y el vino de mesa4.

Por otra parte, la adolescencia es un período de transición de la vida en los seres humanos entre la infancia y la vida adulta, caracterizada por cambios biológicos, psicológicos y sociales ${ }^{5}$. En esta etapa los adolescentes no pueden ser vistos como personas dependientes, pero tampoco pueden ser considerados competentemente adultos para poder tomar decisiones, aceptar responsabilidades y asumir obligaciones. Por lo tanto, la adolescencia es una etapa natural del desarrollo, con carácter universal y abstracto, percibida como una fase difícil, crítica y preocupante caracterizada por conflictos naturales ${ }^{6}$, tales como: organización de tiempo y espacio, apreciación emocional, intercambio interpersonal, enfrentamiento a diversas situaciones, interés sexual, integración al grupo de pares, desarrollo profesional y una autoimagen social ${ }^{7}$.

A medida que los adolescentes viven y experimentan las nuevas formas de hacer las cosas, reclaman con inquietud su independencia; cuestionándose sobre sí mismos y de las complejidades y los matices de la vida. Durante este período los adolescentes establecen su independencia emocional y psicológica, aprenden a entender y vivir su sexualidad y a considerar su papel en la sociedad del futuro, es un proceso gradual, pero en ocasiones perturbador ${ }^{8}$. Se ha identificado que los y las adolescentes pueden sentirse desilusionados y decepcionados en un determinado momento y, poco tiempo después, eufórico y optimista.

También hay que considerar que aspectos familiares, culturales y sociales han incitado a millones de adolescentes a asumir funciones y responsabilidades propias de adultos a tan corta edad, llevándolos a presentar malestar emocional ${ }^{9}$. Bajo esta misma 
línea el malestar emocional puede definirse como el conjunto de sensaciones subjetivas que percibe una persona de que su bienestar sufre un desgaste y que se manifiesta por síntomas inespecíficos, siendo una condicionante para la aparición de enfermedades mentales, sobre todo en personas con vulnerabilidad bio-psico-social ${ }^{10}$.

En esta etapa los adolescentes y jóvenes presentan episodios de enojo, ira, tristeza, depresión, miedo, ansiedad y timidez que pudieran desencadenar conductas nocivas para la salud, tales como el consumo de alcohol, tabaco y otras drogas ${ }^{11}$. Dado que los adolescentes manifiestan que el hecho de consumir alcohol les produce una sensación de placer, tranquilidad e incluso excitación.

A través de este comportamiento buscan sentirse mejor y suplir carencias que poseen a nivel familiar y social en el ámbito afectivo o intelectual, por lo tanto necesita mayor consumo de alcohol hasta llegar a un nivel que puede desorganizar de manera importante su personalidad, generando una serie de disfunciones de su vida real ${ }^{12}$, tomando como referente lo anteriormente expuesto se estableció como objetivo determinar la relación y el efecto del malestar emocional en el consumo de alcohol de los adolescentes.

\section{MÉTODO}

Estudio de tipo cuantitativo de corte transversal, descriptivo y predictivo ${ }^{13}$ llevado a cabo durante el mes de enero de 2018. La población estuvo conformada por 827 adolescentes de una institución pública de educación secundaria de Ciudad del Carmen Campeche, México. Se realizó un muestreo probabilístico, el tamaño de muestra fue calculada con un nivel de significancia de .04, una potencia del $90 \%$ y un coeficiente de correlación alterno de .08, considerando una tasa de no respuesta del $5 \%$, obteniendo una muestra final de 200 sujetos $^{14}$.

Como instrumentos de recolección de datos se utilizó un cuestionario sobre datos sociodemográficos y antecedentes del consumo de alcohol, el cual recopila biológicos (edad y sexo), sociales (ocupación y datos familiares) y características del consumo de alcohol (prevalencias, edad de inicio del consumo de alcohol, numero de bebidas y tipo de bebida).

En relación al malestar emocional, se utilizó el dominio 1 del Cuestionario de Factores de Riesgo y de Protección para el Consumo de Drogas en Jóvenes ${ }^{15}$ diseñado por 
un Grupo de Investigadores en Adicciones del Departamento de Enfermería Psiquiátrica y Ciencias Humanas de la Escola de Enfermagem de Ribeirão Preto de la Universidade de São Paulo (USP). El cual está constituido por 12 reactivos dicotómicos, donde $0=$ No y $1=$ Si, para su interpretación puntuaciones de 0 a 4 indican ausencia de malestar emocional y de 5 a 12 presencia del mismo, este dominio obtuvo un Alpha de Cronbach de .84, lo cual se considera aceptable.

Se han respetado todos los aspectos éticos en investigación con seres humanos, iniciando la recolección de datos solamente después aprobación del proyecto por parte del Departamento de Investigación y Posgrado de la Universidad Autónoma del Carmen bajo el registro FCS/2DOP2017/03, además de la autorización de los directivos de la institución educativa donde se llevó a cabo el estudio. Para la selección de los participantes se solicitaron las listas de grupos de alumnos por semestre y se realizó la selección de manera aleatoria, posteriormente se visitó a los participantes dentro de su horario de clase para invitarles a participar en el estudio, quienes decidieron participar se les hizo entrega de un consentimiento informado para padre o tutor, así como un asentamiento informado dado que se trata de menores de edad, en todo momento se garantizó la confidencialidad, el anonimato y la libertad de participación.

Cabe mencionar que el estudio se apegó a lo dispuesto en la Ley General de Salud en Materia de Investigación para la Salud en Seres Humanos, Capítulo I de los Aspectos Éticos de la Investigación en Seres Humanos y Capitulo III de la Investigación en Menores de Edad o Incapaces ${ }^{16}$.

Los datos se capturaron y analizaron en el programa estadístico Statistical Package for the Social Sciences (SPSS) versión 23.017, se obtuvieron frecuencias y proporciones para las variables categóricas, así como tablas de contingencia complementadas con la Prueba de Ji Cuadrada, además se realizó análisis inferencial por medio del coeficiente de correlación de Spearman y Modelos de Regresión Logística Simple que permitieron explicar la asociación de las variables en estudio.

\section{RESULTADOS}

En relación al perfil sociodemográfico, los adolescentes presentaron una media de edad de 13.24 años ( $D E=2.11 ; 11-16)$ y el sexo masculino (51\%). Cabe mencionar que el $93.5 \%$ de los adolescentes se dedican a estudiar y un 6.5\% estudia y trabaja al mismo 
tiempo, siendo el comercio la principal actividad económica desempeñada, por lo que respecta al ámbito familiar el $73.5 \%$ de los adolescentes vive con ambos padres y el $19.5 \%$ solo con la madre.

Por lo que se refiere a las prevalencias del consumo de alcohol se identificó que el $19.5 \%$ de los adolescentes manifestaron haber ingerido alguna bebida alcohol en algún momento de su vida, el $14 \%$ en el último año, $3 \%$ en el último mes de la aplicación de la encuesta y el $1 \%$ en los últimos siete días; así mismo el $66.7 \%$ ingiere cerveza y el $22.3 \%$ bebidas preparadas.

En cuanto a la escala de malestar emocional, se observó que el $68.5 \%$ señala sentirse relajado, 56\% pasa fácilmente de la tristeza a la alegría o al revés, 53\% manifestó sentirse constantemente aburrido, 43.5\% tiene pensamientos repetitivos, y el $44 \%$ se pone triste fácilmente (Tabla 1).

Además, el malestar emocional por sexo, presento diferencias significativas en los reactivos: "me irrito con facilidad" $\left(x^{2}=7.228, p=.007\right)$ y "me pongo triste con facilidad" ( $\left.x^{2}=11.465, p=.001\right)$, donde es mayor en el sexo femenino en relación al masculino.

Al categorizar el malestar emocional el 36\% de los adolescentes se encuentran sin malestar y el $64 \%$ con malestar emocional; al relacionarlo con los tipos de prevalencia se encontraron diferencias significativas $(p=>.05)$ con el consumo de alcohol alguna vez en la vida y el último año, donde es mayor en aquellos adolescentes que si han consumido alcohol y que si presentan malestar emocional (Tabla 2).

Al analizar el consumo de alcohol alguna vez en la vida por los ítems de la escala de malestar emocional, se obtuvieron diferencias significativas con los reactivos siguientes: "me siento muy afectado por mis problemas" $\left(x^{2}=6.46, p=.011\right)$, "tengo pensamientos que me fastidian" ( $\left.x^{2}=4.127, p=.041\right)$, "paso fácilmente de la tristeza a la alegría o viceversa" ( $\left.x^{2}=8.608, p=.003\right)$ y "me pongo triste fácilmente" $\left(x^{2}=7.946\right.$, $p=.005)$, donde es mayor en malestar emocional en aquellas personas que no han consumido alcohol.

Por lo que se refiere a la relación del malestar emocional y el consumo de alcohol de los adolescentes, se observó una relación positiva y significativa ( $r s=.473 . p=.003)$, lo cual indica que a mayor malestar emocional mayor será la ingesta de bebidas alcohólicas consumidas en un día típico. Para analizar el efecto de malestar y el consumo de alcohol (prevalencias) se realizó un modelo de regresión lineal simple 
(Tabla 3), teniendo como variable independiente el malestar emocional y la dependiente el consumo de alcohol, donde el Modelo fue significativo para el consumo de alcohol en la última semana $(p=.001, f=10.787, g l=1)$ con una varianza explicativa del $5.2 \%$.

Tabla 1. Frecuencias y proporciones del malestar emocional en adolescentes.

\begin{tabular}{lcccc}
\hline \multicolumn{1}{c}{ Malestar emocional } & \multicolumn{3}{c}{ Sí } & \multicolumn{3}{c}{ No } \\
\cline { 2 - 5 } & $\mathrm{f}$ & $\%$ & $\mathrm{f}$ & $\%$ \\
\hline 1. Constantemente me siento aburrido & 106 & 53.0 & 94 & 47.0 \\
2. Me siento muy afectado por mis problemas. & 59 & 29.5 & 141 & 70.5 \\
3. Tengo problemas para dormir o me despierto con & 67 & 33.5 & 133 & 66.5 \\
facilidad. & 87 & 43.5 & 113 & 56.5 \\
4. Tengo pensamientos repetitivos. & 76 & 38.0 & 124 & 62 \\
5. Tengo dificultad para relajarme. & 79 & 39.5 & 121 & 60.5 \\
6. Tengo pensamientos que me fastidian. & 112 & 56.0 & 88 & 44.0 \\
7. Paso fácilmente de la tristeza a la alegría o al & 79 & 39.5 & 121 & 60.5 \\
revés. & 137 & 68.5 & 63 & 31.4 \\
8. Me irrito con facilidad. & 63 & 31.5 & 137 & 68.5 \\
9. Me relajo. & 62 & 31 & 138 & 69 \\
10. Me siento angustiado con frecuencia. & 88 & 44 & 112 & 56 \\
11. Me tensiono fácilmente. & & &
\end{tabular}

Nota: $f=$ frecuencia, $\%=$ porcentaje, $n=200$.

Tabla 2. Prueba de Chi Cuadrada para el consumo de alcohol por malestar emocional.

\begin{tabular}{lcccccc}
\hline \multirow{2}{*}{ Consumo de alcohol } & & Sin Malestar & Con malestar & \multirow{2}{*}{$x^{2}$} & \multirow{2}{*}{$P$} \\
\cline { 2 - 4 } Alguna vez en la vida & No & 66 & $\mathrm{f}$ & 95 & \multirow{2}{*}{8.937} & \multirow{2}{*}{.003} \\
\multirow{2}{*}{ En el último año } & Si & 6 & 33 & & \\
\multirow{2}{*}{ En el último mes } & No & 67 & 105 & 4.651 & .031 \\
\multirow{2}{*}{ En la última semana } & Si & 5 & 23 & & \\
& No & 72 & 122 & 3.47 & .052 \\
\hline
\end{tabular}

Nota: $f=$ frecuencia, $x^{2}=$ Valor de Chi Cuadrada, $p=$ significancia.

Tabla 3. Modelo de Regresión Lineal Simple pata el malestar emocional sobre el consumo de alcohol en la última semana.

\begin{tabular}{|c|c|c|c|c|c|c|}
\hline & $\begin{array}{l}\text { Suma de } \\
\text { Cuadrados }\end{array}$ & $g l$ & $\begin{array}{c}\text { Cuadrado } \\
\text { Medio }\end{array}$ & $f$ & $P$ & $\mathrm{R}^{2}$ \\
\hline Regresión & .102 & 1 & .102 & 10.787 & .001 & 5.2 \\
\hline Residual & 1.878 & 198 & .009 & & & \\
\hline \multirow[t]{2}{*}{ Total } & 1.980 & 199 & & & & \\
\hline & & \multicolumn{2}{|c|}{$\begin{array}{l}\text { Coefientes no } \\
\text { estandarizados }\end{array}$} & $\begin{array}{c}\text { Coeficiente } \\
\text { Tipificado }\end{array}$ & & \\
\hline \multicolumn{2}{|l|}{ Vari } & $B$ & $D E$ & $B$ & $T$ & $P$ \\
\hline & nte & -.034 & .015 & & -2.250 & .026 \\
\hline \multicolumn{2}{|c|}{ Malestar Emocional } & .009 & .003 & .227 & 3.284 & .001 \\
\hline
\end{tabular}

Nota: $g l=$ grados de libertad, $f=$ fuerza, $p=$ significancia, $R^{2=}$ varianza explicada 


\section{DISCUSIÓN}

Los resultados de la presente investigación permitieron determinar la relación y el efecto del malestar emocional sobre el consumo de alcohol de los adolescentes de secundaria. Se identificó una prevalencia global de 19.5\% y lápsica del 14\%, lo cual es inferior a lo reportado en adolescentes de Ciudad Nezahualcóyotl México ${ }^{18}$ y de Coatzacoalcos Veracruz ${ }^{19}$. Esto pudiera estar vinculado a la influencia de factores de protección en los adolescentes de forma individual, familiar, grupal y de la propia comunidad donde el adolescente crece y se desenvuelve, lo cual favorece el desarrollo humano, el mantenimiento o la recuperación de la salud, contrarrestando los posibles efectos de los factores y conductas de riesgo y por ende la vulnerabilidad, característica principal de esta etapa.

Los adolescentes manifestaron sentirse relajados, aburridos y pasar fácilmente de la alegría a la tristeza, además de irritarse y ponerse triste con facilidad, siendo predominando este tipo de emociones en las mujeres, siendo similar a lo reportado en adolescentes de secundaria de la Ciudad de México, donde los problemas psicológicos y sociales desencadenan una seria de emociones y sentimientos que pudieran ocasionar trastornos psicológicos tales como depresión, baja autoestima, pensamientos o ideas suicidas y suicidios ${ }^{20}$. Así también se ha señalado que las mujeres viven y expresan de manera muy peculiar las emociones en relación a los hombres, especialmente las relacionadas con el miedo y el disgusto, donde estas emociones influyen directamente en la memoria de las personas, por lo tanto, cuanto más emocional es una situación, más probable es que se recuerde ${ }^{21}$.

El 64\% de los adolescentes presenta malestar emocional, lo cual concuerda con investigaciones internacionales ${ }^{22,23}$ al mencionar que la etapa de la adolescencia constituye el tiempo de vida de un individuo donde se adquieren y desarrollan nuevas habilidades, donde la vida social suele ser complicada, las relaciones familiares son cambiantes, se aplican los valores y actitudes adquiridos durante la infancia, además de presentarse cuestionamientos sobre identidad personal y del futuro, que llega a ser incierto en esta etapa. Estos cambios de tipo afectivo, fisiológico, psicológico, pudieran llegar a alterar la percepción del tiempo, espacio, la manera de ver el mundo y de relacionarse con los otros. 
Al analizar la prevalencia global del consumo de alcohol por los reactivos de la escala de malestar emocional, se obtuvieron diferencias significativas, siendo mayor el malestar emocional en aquellos adolescentes que no han consumido alcohol. Estos hallazgos concuerdan con lo estipulado por un grupo de investigadores de Valencia España que señalan que los problemas que presentan los adolescentes no son atribuibles a un factor en especificó, sino más bien el contexto donde se desarrolla, tales como el ámbito familiar y escolar que juegan un papel crucial en el ajuste psicológico del individuo y que pudiera ocasionar problemas de salud mental tales como depresión, ansiedad y estrés, baja autoestima académica/escolar o los problemas de integración social y victimización entre iguales ${ }^{24}$.

Se evidenció una relación y efecto positivo del malestar emocional y el consumo de alcohol de los adolescentes. En este sentido se ha documentado que al parecer los adolescentes y jóvenes ingieren bebidas alcohólicas como un mecanismo que les ayude a recuperar el equilibrio emocional, pasando de un estado negativo (antes de consumir) a uno positivo (durante y después de ingerir alcohol), obteniendo como resultado un bienestar personal, este bienestar junto con la relajación y la reducción del estrés, parecen ser motivos para ingerir alcohol ${ }^{25}$. Es así que las emociones durante esta etapa suelen ser muy intensas, donde los jóvenes se vuelven más reactivos y menos tolerantes, y en raras ocasiones agresivos ${ }^{26}$. Además de sentimientos de omnipotencia e inmortalidad que pueden llevarle a conductas arriesgadas como el consumo de alcohol ${ }^{27}$, tabaco y otras drogas $^{28}$, los cuales pudieran tener un efecto negativo sobre el desarrollo del cerebro principalmente sobre la corteza prefrontal y en otras áreas corticales y subcorticales, esenciales en las funciones ejecutivas del cerebro ${ }^{29}$.

\section{CONCLUSIÓN}

Los resultados del estudio permitieron determinar la relación y el efecto del malestar emocional y el consumo de alcohol en adolescentes de educación secundaria de Ciudad del Carmen Campeche, México. Estos hallazgos ponen de manifiesto por un lado el consumo de alcohol en esta población, caracterizada por cambios a nivel biológico, psicológico y social; donde el consumo de alcohol pudiera ocasionar múltiples y daños significativos. 
Por otro lado, el malestar emocional representa un fenómeno presente en la población adolescente que pudiera desencadenar una serie de comportamientos nocivos, tal como la ingesta del alcohol, como una forma de mitigar ese conjunto de emociones y sentimientos negativas y/o positivas característicos de esta etapa.

Así también, constituye un área de oportunidad para que los profesionales de la salud encaminen acciones específicas en este grupo vulnerable de la sociedad. Por parte del profesional de enfermería el diseño e implementación de intervenciones educativas para fortalecer los factores de protección y contrarrestar los factores de riesgo a los cuales se encuentran expuestos los adolescentes, así como el punto de partida para próximos estudios de corte cuantitativo y cualitativo que permitan la comprensión a profundidad del fenómeno.

\section{REFERENCIAS}

1. Organización Mundial de la Salud. Estrategia mundial para reducir el uso nocivo del alcohol. [Internet]. 2010. [acceso 02 de Febrero de 2018]. Disponible en https: //www.unodc.org/unodc/es/drug-prevention-and-treatment/index.html

2. Organización Mundial de la Salud. Alcohol. [Internet]. 2014. [acceso 02 de Febrero de 2018]. Disponible en http: / / www.paho.org/hq/index.php?option=com_topics\&view=article\&id=197\&ltemid=4 $0861 \&$ lang=es.

3. Organización Panamericana de la Salud. Regional Status Report on Alcohol and Health in the Americas. [Internet]. 2015. [acceso 03 de Febrero de 2018]. Disponible en http: / / www.paho.org/hq/index.php?option=com_content\&view=article\&id=11108\%3A20 15-regional-report-alcohol-health\&catid=1893\%3Anews\&ltemid=41530\&lang=es

4. Instituto Nacional de Psiquiatría Ramón de la Fuente Muñiz, Comisión Nacional Contra las Adicciones, Secretaría de Salud. Encuesta Nacional de Consumo de Drogas en Estudiantes 2014: Reporte de Alcohol. [Internet]. 2014. [acceso 03 de Febrero de 2018]. Disponible en: http://www.conadic.salud.gob.mx/pdfs/investigacion/ENCODE_ALCOHOL_2014.pdf 5. Silva IL. Adolescentes y percepción del sí mismo: la construcción de una imagen realista de la adolescencia desde la familia y la escuela. Revista científica electrónica de Educación y Comunicación en la Sociedad del Conocimiento. 2011; 9 (11): 233-252. 
6. Balistieri SA, Tavares MMC. The importance of social-emotional support in adolescents and young adults with chronic disease: a literature review. Enferm glob. 2013; 30: 388-398.

7. Meneses-Heredia A, Bello-Davila Z. Reaction of Adaptation in Adolescents and its Link with the Emotional Capacities. Rev Educación y Desarrollo. 2013; 26: 29-37.

8. Urbiola I, Estevez A, Iruarrizaga I, Jauregui P. Dependencia emocional en jóvenes: relación con la sintomatología ansiosa y depresiva, autoestima y diferencias de género. Ansiedad estrés. 2017; 23(1):6-11.

9. Fondo de las Naciones Unidas para la Infancia (UNICEF). Adolescenia una etapa fundamental. [Internet]. 2002. [acceso 03 de Febrero de 2018]. Disponible en: https://www.unicef.org/ecuador/pub_adolescence_sp.pdf

10. Gorn SB, Reyes JG, Solano NS, Pérez PB, Mellor-Crummey L, Saínz MT. Exploración del malestar emocional expresado por mujeres que acuden a centros de atención primaria de la Ciudad de México. Un estudio cualitativo. Salud ment. 2014; 37:313-319.

11. Poncela AMF. Adolescencia, crecimiento emocional, proceso familiar y expresiones humorísticas. Educar. 2014; 50(29):445-66.

12. Ordoñez MA, Ortega LM. Factores asociados al consumo de alcohol en estudiantes de los dos primeros años de carrera universitaria. Liberabit. 2008; 14(14):49-61.

13. Burns N, Grove SK. The practice of nursing research: appraisal, synthesis and generation of evidence. Missouri: Saunders; 2009.

14. Elashoff J, Dixon JW, Crede KM, Fotheringham N. nQuery Advisor (Programa de Computadora). Boston MA: Release 4.8; 2000.

15. Morales BN, Plazas M, Sanchez R, Ventura CAA. Factores de riesgo y de protección relacionados con el consumo de sustancias psicoactivas en estudiantes de enfermería. Rev Latino-Am Enferm. 2011; 19(spe): 673-83.

16. Secretaria de Salud. Reglamento de la Ley general de salud en materia de investigación para la salud; 1987 [citado 06 de febrero 2018].Disponible en: http://www.salud.gob.mx/unidades/cdi/nom/compi/rlgsmis.html

17. SPSS ${ }$ Statistics for Windows (Versión 23.0.0) [Software de computación]. Armonk, NY: IBM Corp; 2012.

18. González FM, Jiménez MC, Barajas CJP, Franco MLA. El consumo de alcohol en adolescentes de una secundaria de Ciudad Nezahualcóyotl Frecuencia y características sociales, 2015. Vertientes. 2015; 18(1):8-15. 
19. Tegoma-Ruiz VM, Cortaza-Ramírez L. Prevalencia del consumo de alcohol en adolescentes de una secundaria de Coatzacoalcos, Veracruz. Enferm Univ. 2016; 13(4):239-45.

20. Moyeda IXG, Velasco AS, Ojeda, FJR.Galicia MIX, Sánchez VA, Robles OFJ. Factores asociados a la depresión en adolescentes: Rendimiento escolar y dinámica familiar. An psicol. 2009; 25(2):227-40.

21. Spalek K, Fastenrath M, Ackermann S, Auschra B, Coynel D, Frey J, et al. SexDependent Dissociation between Emotional Appraisal and Memory: A Large-Scale Behavioral and Study. J Neurosci. 2015; 35(3):920-35.

22. Silva A, Gálviz L. Silencio y grito de la adolescencia. Fermentum. 2010; 20(58):187-206.

23. Awuapara-Flores S, Vargas-Machuca MV. Características bio-psicosociales del adolescente. Odontol Pediatr. 2013; 12(2):119-28.

24. Lopez EE, Ochoa GM, Olaizola, JH. El rol de la comunicación familiar y del ajuste escolar en la salud mental del adolescente. Salud ment. 2005; 28(4):81-9.

25. Delgado JRP. Exploración de los motivos para consumir alcohol en adolescentes. Psicol iberoam. 2012; 20(1):29-39.

26. Escobedo PAS, Cuevas MIC, Cuervo AAV, García JV. Percepciones de adolescentes mexicanos acerca del adolescente y el hombre y la mujer ideales. Ensen investigación psicol. 2012; 17(2):283-93.

27. Diz JLI. Adolescencia. Aspectos físicos, psicológicos y sociales. An Pediatr Contin. $2014 ; 12(1): 42-6$.

28. Sánchez-Ventura GJ. Prevención del consumo de alcohol en la adolescencia. Rev Pediatr Aten Primaria. 2012; 14(56):335-42.

29. García FDR, Ruiz MLS, Alzina RB. Consumo de alcohol en la adolescencia. Consideraciones médicas y orientaciones educativas. Salud ment. 2014; 37(3):255-60.

Conflito de interesses: Os autores declaram não haver conflito de interesses.

Participação dos autores: Os autores declaram que participaram de todas as etapas do estudo (concepção, desenvolvimento do estudo, redação e revisão).

Como citar este artigo: Terrero JYT, Cisneros MAL, Telumbre LEP, Linares ODV, Obregon RT, Almanza SEE. Relación del malestar emocional y el consumo de alcohol en adolescentes. Journal Health NPEPS. 2018; $3(1): 38-50$. 
Submissão: 18/02/2018

Aceito: 20/06/2018

Publicado: $30 / 06 / 2018$ 